Bazzini, D. G. \& Shaffer, D. R. (1997). What do you look for in a prospective date?: Reexamining the preferences of men and women who differ in self-monitoring propensities. Personality and Social Psychology Bulletin, 23(6): 605-616. (June 1997) Published by Sage (ISSN: 0146-1672).

\title{
What do you look for in prospective date? Reexamining the preferences of men and women who differ in self-monitoring propensities
}

\author{
Doris G. Bazzini and David R. Shaffer
}

Males and females who differed in self-monitoring propensities indicated their preferences for an evening date by ranking the desirability of nine opposite-sex targets who were varied in attractiveness and personality. In contrast to past research, criteria used to evaluate dating partners were not influenced by participants' self-monitoring propensities. Results provided little evidence that dating orientations of high and low self-monitors vary systematically when partners are selected from a broad, representative array of alternatives. Consistent with past research, men weighed attractiveness cues more heavily than did women; women attached more weight to dispositional information than men did. Yet, internal analyses revealed that the dating orientations of male and female participants were really more similar than different: Both the looks and the character of prospective dating partners were highly salient to men and women, who were most interested in dating individuals who maximized total outcomes across both dimensions.

Embedded within the burgeoning literature on the formation, maintenance, and dissolution of interpersonal relationships is a body of evidence implying that people who differ in selfmonitoring propensities adopt distinctly different orientations toward dating relationships (Snyder \& Simpson, 1984), particularly with regard to the use of physical attractiveness as a criterion for date selection (Snyder, Berscheid, \& Glick, 1985). Snyder et al. (1985, Experiment 1), for example, found that high-self-monitoring individuals paid significantly more attention to the physical appearance of potential dating partners than did low-self-monitoring individuals, who, in 
turn, devoted relatively more attention to the targets' dispositional attributes (e.g., sociability, openness, etc.). Moreover, participants seemed quite aware of the differences in their attentional allocations: $77 \%$ of the high-self-monitoring individuals stated that they were using physical attractiveness information as their most important criterion for evaluating the prospective dating targets, whereas $75 \%$ of the low-self-monitoring individuals listed internal attribute information (i.e., personality) rather than attractiveness as their primary criterion for evaluating prospective dates.

The results of this initial experiment were quite congruent with the premises of self-monitoring theory. Allegedly, high-self-monitoring individuals, who are known to be especially concerned about the impressions they create, will attend closely to their partners' physical appearance because associating with attractive others should create a favorable impression. By contrast, low-self-monitoring individuals, who are known to value acting in accordance with their own true attitudes and values, are thought to attend more to their partners' inner qualities because partners with particular values, attitudes, or personalities are more likely to facilitate the low selfmonitor's need for self-expression (Snyder et al., 1985). In a second experiment, Snyder et al. (1985) led high- and low-self-monitoring men to believe that they were participating in an actual dating experiment and would be asked to go on an evening date with whomever of two prospective partners they happened to select. One of the stimulus females was highly attractive but was characterized as having several undesirable personality traits (e.g., self-centeredness, moodiness), whereas the second stimulus person (SP) was rather plain and unattractive but displayed highly desirable dispositional attributes (e.g., sociability, good sense of humor). The choices participants made dovetailed nicely with the results of the first experiment: $69 \%$ of the high-self-monitoring participants selected the attractive woman with an undesirable personality, whereas $81 \%$ of the low-self-monitoring individuals chose the unattractive woman with the desirable personality (see also Glick, 1985).

Apparently, the differing criteria that high- and low-self-monitoring individuals use to select their own dating partners also affect their beliefs about how other people should choose their partners. Glick, DeMorest, and Hotze (1988), for example, gave participants photographs and personality information about 5 men and 5 women and asked them to form, from these 10 individuals, the five couples that they thought would be most compatible. For each of the 5 male SPs, there was 1 female target of a similar level of rated physical attractiveness and a second female target whose personality was similar to his own. The results were clear: High-selfmonitoring individuals were more likely to match couples on the basis of physical attractiveness than were low-self-monitoring individuals, who, instead, were more inclined to use personality information when making their matches.

\section{Does the Literature Overstate the Case?}

From the existing literature, then, one might well infer, as Snyder (1987) has, that "people initiate romantic relationships on very different bases--exterior appearances for high selfmonitors and interior qualities for low self-monitors" (p. 73). Indeed, this conclusion is widely and uncritically reported in contemporary textbooks on personality (e.g., Cloninger, 1996) and social 
psychology (e.g., Horowitz \& Bordens, 1995; Myers, 1996). Yet, there are three observations-one empirical and two conceptual--that cause us to question the magnitude and generality of these findings. On the empirical front, Bazzini and Shaffer (1995) conducted a conceptual replication of the couple-matching task reported by Glick et al. (1988) and, indeed, found that couples matched by high-self-monitoring individuals varied significantly less in attractiveness and significantly more in personality than those matched by low-self-monitoring individuals. Yet, on an absolute basis, the couples that participants matched deviated substantially from those one would have expected had they been paired mainly on their levels of physical attractiveness or on similarities in personality. For example, the average attractiveness difference scores of pair members matched by high-self-monitoring individuals deviated substantially from the "best" (or closest) attractiveness matches they could have made--so much so, in fact, that they were actually closer to the attractiveness difference scores of couples matched by low-self-monitoring participants than to the "ideal" attractiveness matches. Moreover, the average personality difference scores of pair members matched by low-self-monitoring individuals were more similar in magnitude to those of couples matched by high-self-monitoring individuals than to those that would have resulted from the best (or closest) matching of SPs on the basis of personality information. One interpretation of these findings is that both high- and low-self-monitoring individuals were carefully considering attractiveness and personality information when matching couples, almost as if they were seeking to minimize as best they could the differences their couples would display on both dimensions.

Might high- and low-self-monitoring individuals adopt a similar strategy when selecting their own dating partners? Unfortunately, the previous literature (Glick, 1985; Snyder et al., 1985) cannot tell us, or participants were required to choose between SPs for whom attractiveness information and personality information were perfectly confounded (i.e., an attractive but characterologically undesirable SP vs. an unattractive SP with a desirable personality). Thus, prior research does imply that high-self-monitoring individuals are often willing to sacrifice personality for attractiveness, and that low-self-monitoring individuals may often be inclined to sacrifice attractiveness for a desirable personality, when choosing among extremes that constrain them to make such sacrifices. But rarely are people faced with such extreme constraints, and it is possible, even likely, that existing research seriously overdramatizes differences in the dating orientations of high- and low-self-monitoring individuals. In the natural environment, people looking to initiate dating relationships can often select their partners from a much broader array of targets--that is, from individuals who vary considerably in their levels of physical attractiveness and the desirability of their dispositional attributes. Under these circumstances, we believe that virtually all individuals--high and low self-monitors alike--would carefully consider both attractiveness information and personality characteristics in selecting their dating partners, seeking to maximize, as best they can, their outcomes on both dimensions.

What we are proposing here in offering our maximization of outcomes hypothesis is an alternative viewpoint that challenges the presumption that high- and low-self-monitoring individuals "adopt systematically different approaches to gathering, weighing, and acting on information about potential [dating] partners" (Snyder et al., 1985, p. 1436, emphasis added). When evaluating a broader array of opposite-sex targets as prospective dates, it is entirely 
possible that high-self-monitoring participants will weigh physical attractiveness information somewhat more heavily and personality information somewhat less so than their low selfmonitoring counterparts. Yet, the maximization hypothesis clearly implies that all participants should be willing to make some very noteworthy concessions along their more heavily weighted dimension (e.g., high-self-monitoring individuals favoring a moderately attractive person with a highly desirable personality over a highly attractive SP with a very undesirable personality) whenever such a choice would maximize their outcomes across both dimensions. Thus, in the present project--in which attractiveness cues and the desirability of dispositional information are orthogonally varied (rather than perfectly confounded)--participants were asked to evaluate as prospective dating partners an array of opposite-sex targets. Our purposes in so doing were (a) to re-examine the dating preferences of high- and low-self-monitoring individuals when perusing targets from a less constraining set of options, with (b) an eye toward testing the maximization of outcomes hypothesis outlined above.

A secondary objective of the present research was to correct what appears to be another potentially serious shortcoming of existing research: a heavy reliance on men as research participants (Glick, 1985; Snyder et al., 1985).(1) Indeed, there is an emerging empirical consensus (i.e., Buss, 1989; Feingold, 1990, 1992; Fischer \& Heesacker, 1995; but see Graziano, Jensen-Campbell, Shebilske, \& Lundgren, 1993) that women weigh demographic and dispositional information more heavily and attractiveness cues less heavily than men do when evaluating romantic partners. Often, these gender differences in partner/mate selection are explained in evolutionary terms (with men allegedly being attracted to young, attractive females to maximize their motivation to and likelihood of producing offspring, and with women paying less attention to attractiveness and more attention to such prospective partner attributes as cooperation/pro-social orientations to maximize the survival or reproductive prospects of their potential offspring; cf. Buss \& Schmitt, 1993; Feingold, 1992; Jensen-Campbell, Graziano, \& West, 1995; Trivers, 1972), although several sociocultural explanations for such sex differences have also been offered (see Hatfield \& Rapson, 1996, for an excellent review). Regardless of why these gender differences may have emerged, they raise the intriguing possibility that any variations in the dating orientations of high- and low-self-monitoring individuals may be stronger for men than for women. The couple-matching study reported by Glick et al. (1988) did include participants of both sexes and reported no gender differences (nor a Gender x Self-monitoring interaction) in participants' use of either attractiveness or personality information to match compatible couples. However, Bazzini and Shaffer (1995) found significant gender effects: Couples matched by female participants varied significantly less in personality and significantly more in attractiveness than did couples matched by male participants. So research implying that women may be more dispositionally oriented (and less attractiveness oriented) than men when evaluating opposite-sex targets alerts us to the possibilities that (a) gender may play a strong role in moderating dating choices, so much so that (b) any differences in the dating orientations of high- and low-self-monitoring individuals may be limited to (or more readily apparent among) our male participants. Nevertheless, we strongly suspect that when evaluating prospective dates from a broad array of options, both men and women will carefully consider both attractiveness and dispositional attributes, seeking to maximize, as best they can, their outcomes on both dimensions. 


\section{METHOD}

\section{Participants and Design}

Participants were 50 male and 50 female introductory psychology students who volunteered to take part in what was advertised as a study of dating behavior and entitled "Formation of Dating Relationships." All participants had earlier completed the Self-Monitoring Scale (Snyder \& Gangestad, 1986) in a group testing session and were subsequently classified as high ([is greater than or equal to] 9) or low ([is less than or equal to] 8) in self-monitoring propensities based on a median split of the distribution of scores for the larger group-testing sample. Thus, the design was a 2 (subject gender) $\times 2$ (self-monitoring) factorial.

\section{Procedure}

Each participant was run individually by a female experimenter who was unaware of the participant's self-monitoring propensities. The participant was seated at a table across from the experimeter, who explained that she was in the initial phase of a dating study that would be pairing couples for purposes of going out on one or more evening dates. It was further explained that the procedure would require each participant to examine information about nine oppositesex SPs who, like themselves, had volunteered to participate in a dating study. Specifically, the task was to rank order the nine SPs with respect to their desirability as prospective dating partners. Allegedly, these rankings were one of the more important pieces of information that the experimenter would consult in her attempt to match compatible couples for the upcoming dates. To further encourage participants to carefully peruse the nine SPs and to take their rankings seriously, each was told that he or she would have an opportunity to meet briefly with two or three of his or her higher ranked SPs in the very near future. After questioning participants to ensure that they understood the purposes of the study and giving them an opportunity to withdraw (which no one did), the experimenter distributed the stimulus materials.

Stimulus materials. The participant then received nine 5 in. $x 7$ in. index cards, each of which was identified by a number and contained (a) a small yearbook-like photograph of an oppositesex SP and (b) information about three of the SP's most noteworthy personality characteristics. Attractiveness cues and desirability of personality information were orthogonally varied so that each of nine SPs displayed one of three possible levels of attractiveness (high, moderate, or low) and one of three personality profiles (high, intermediate, or low in desirability).

To ensure that attractiveness cues were varied as intended, a pretest sample of 20judges (10 males and 10 females) rated the attractiveness of each photograph on a 12-point scale ( $1=$ very unattractive, 12 = very attractive). These attractiveness scores were then summed and averaged to obtain a single score for each SP. The mean attractiveness ratings were as follows: For the highly attractive condition, Ms = 9.2 for male SPs and 9.4 for female SPs; for the moderately attractive condition, Ms $=6.1$ for male SPs and 5.9 for female SPs; for the unattractive condition, Ms $=3.6$ for both the male and the female SPs. A Tukey's studentized range test revealed that highly attractive SPs of each gender were rated significantly more 
attractive ( $p$ [is less than] .05) than their moderately attractive counterparts, who, in turn, were perceived as more attractive ( $p$ [is less than] .05) than the unattractive SPs.(2)

Anderson's (1968) trait likableness ratings were employed in constructing SPs' personality profiles. The highly desirable profiles each consisted of three traits for which the mean likability ratings were greater than 5.0 on a 7-point scale. The nine traits used in constructing these profiles were as follows: honest, thoughtful, dependable, understanding, witty, friendly, trustworthy. good-natured, and cheerful. The three profiles of intermediate desirability each consisted of three traits with likableness ratings that ranged between 3.0 and 4.4. The traits used to construct these profiles were as follows: practical, easygoing, impulsive, orderly, conservative, candid, strong-minded, unpredictable, and nonconforming. Finally, the undesirable profiles each consisted of three traits that had likableness ratings of less than 2.5. The traits used were as follows: indecisive, pessimistic, short-tempered, cynical, moody, possessive, irritable, stubborn, and insecure. To ensure that personality cues were varied as intended, our pretest sample rated on a 7-point scale the likability of a person who possessed the characteristics described in each of the nine personality profiles. A Tukey's studentized range test revealed that persons described by the highly desirable profiles were perceived as more likable ( $M=5.2, p$ [is less than] .05) than those described by the profiles of intermediate desirability ( $M=3.7)$, who, in turn, were judged more likable ( $p$ [is less than] .05) than those described by the undesirable personality profiles $(M=1.8) .(3)$

Date-ranking measure. After distributing the stimulus materials, the experimenter left the room and allowed the participant 15 min to peruse the information provided and to rank order the nine SPs with respect to their desirability as prospective dates. This rank ordering required the participant to indicate on a rating sheet the identifying number of the SP selected as his or her first choice, second choice, third choice, and so on, until all nine SPs had been ranked.

At the end of the 15-min period, the experimenter returned, collected the participant's dateranking data, and began a funnel-type debriefing to probe for suspicion. Participants' responses to the debriefing indicated that all of them believed the dating study cover story. Special care was then taken to fully explain that it had been necessary to deceive participants about the dating scenario and the impending meeting with opposite-sex SPs to ensure that they would carefully peruse the stimulus materials and take the date-ranking task seriously. Although many participants expressed disappointment at this turn of events, they generally concluded that the deception seemed necessary to obtain valid judgments, and none of them elected to withdraw their data from consideration when given an opportunity to do so. After he or she had been debriefed, the participant was sworn to secrecy, sincerely thanked for his or her participation, and dismissed.

Participants' physical attractiveness. Snyder et al. (1985) have raised the possibility that reliance on attractiveness cues or on personality information as criteria for date selection may depend, in part, on participants' own levels of physical attractiveness (with relatively attractive individuals weighing attractiveness cues more heavily than do less attractive individuals). Even though this alternative participant attractiveness interpretation did not account for the different dating orientations of high-and low-self-monitoring individuals in the Snyder et al. study (i.e., 
high-self-monitoring individuals were judged no more physically attractive than their low selfmonitoring counterparts), we deemed it worth reconsidering in the present project.

When each participant reported to the laboratory, he or she was independently rated for physical attractiveness by three opposite-sex judges who were hidden from view. Each judge was able to see the participant's face and body and used these cues to rate him or her on a 12point attractiveness scale ( $1=$ very unattractive, $12=$ very attractive). Because the ratings were highly reliable across judges (Cronbach's [Alpha] = .86), they were averaged to provide a single attractiveness score for each participant.

\section{RESULTS}

\section{Absolute Weighting of Attractiveness and Personality Information}

To assess the absolute (and relative) emphases participants were placing on attractiveness cues and on personality information when ranking the nine prospective dating partners, we first dummy coded each SP for attractiveness $(2=$ highly attractive; $1=$ moderately attractive; $0=$ unattractive) and for personality information ( 2 = highly desirable personality; 1 = moderately desirable personality; $0=$ undesirable personality). Then, for each participant, an attractiveness weighting score was calculated by multiplying the attractiveness value (i.e., 2, 1, or 0) of his or her first-ranked SP by 9 , the second-ranked SP by 8 , the third-ranked SP by 7 , and so on down the line. Use of this inverse multiplier scheme rests on the assumption that a participant is most interested in dating his or her top choice, next most interested in the second choice, and so on. The resulting cross products were then summed across the nine SPs to provide the participant with an attractiveness weighting score that could range from a high of 63 to a low of 27.(4) Analogous personality weighting scores, which could also range in value from 27 to 63, were calculated for each participant by multiplying the personality value (i.e., 2, 1, 0) of the SP at each rank by the inverse of that rank and summing the cross products across the nine SPs.(5) The resulting attractiveness weighting and personality weighting scores (and the differences between them) were then subjected to a set of 2 (subject gender) $\times 2$ (self-monitoring) analyses.

Because a preliminary analysis revealed that our female participants were judged more physically attractive, on average $(M=7.91$ on a 12 -point scale), than our male participants $(M=$ 7.43), $F(1,96)=4.24, p$ [is less than] .05 , we elected to analyze participants' date-ranking data within the context of 2 (subject gender) x 2 (self-monitoring) ANCOVAs, with participant physical attractiveness as the covariate. The resulting analyses of the attractiveness weighting scores, the personality weighting scores, and the differences between them (i.e., attractiveness weighting minus personality weighting) each produced but one significant outcome--a main effect for participant gender. As illustrated in Table 1, female participants weighted attractiveness cues less heavily, $F(1,95)=5.79, p$ [is less than] .05, and personality information more heavily, $F(1,95)=13.04, p$ [is less than] .001, when ranking the nine SPs than did their male counterparts. Analyses of the difference measure revealed that male participants relied more heavily on attractiveness cues than on personality information when ranking the nine SPS $(M=5.92)$, whereas female participants relied about equally on both types of information $(M=$ - 
0.02), $F(1,95)=10.47, p$ [is less than] .01. Interestingly, none of the main effects for selfmonitoring propensities nor any of the interaction terms approached significance in these analyses (all Fs [is less than] 1. 8, ps [is greater than] .20).

TABLE 1: Mean Attractiveness Weighting and Personality Weighting Scores and Differences Between Attractiveness and Personality Weighting as a Function of Participant Gender and Self-Monitoring Propensities

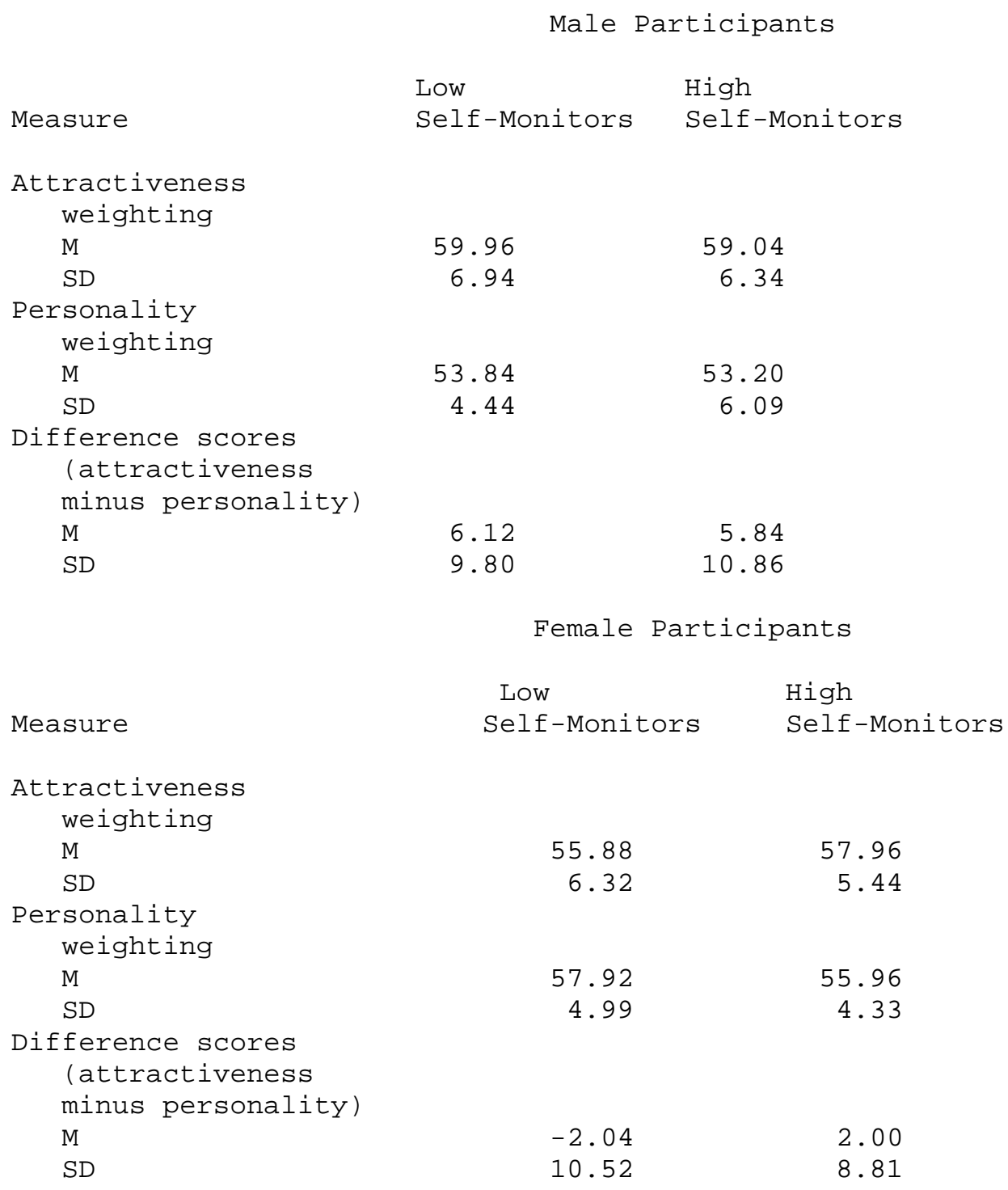

NOTE: Each mean is based on an $n$ of 25.

It should be noted that the pattern of outcomes reported above also emerged and required no qualification when the data were submitted to $2 \times 2$ ANOVAs, without covarying participant 
physical attractiveness. These latter findings seem to rule out the hypothesis that relatively attractive participants would be more likely to weigh attractiveness cues heavily; our female participants, who were judged more attractive than the male participants, were actually less inclined than males to base their ranking of the nine SPs on physical attractiveness information.

Date rankings of self-monitoring extremes. A potentially important point of departure between the present study and that of Snyder et al. (1985) centers on sampling of participants: We used a median split to classify our participants as high or low in self-monitoring, whereas Snyder et al. selected participants who scored [+ or -] .5 standard deviations from the mean score on the Self-Monitoring Scale. Could the fact that our participants were less extreme in their selfmonitoring propensities account for our failure to find any self-monitoring effects on the dateranking measure?

To check on this possibility, we conducted follow-up analyses of the date-ranking data provided by those participants who scored [is less than or equal to] $5(n=23)$ and [is greater than or equal to] $12(n=22)$ on the Self-Monitoring Scale--a subsample that closely approximated the upper and the lower quartiles of our distribution of scores for the self-monitoring dimension. The resulting 2 (participant gender) $\times 2$ (self-monitoring) ANCOVAs of the attractiveness weighting scores, the personality weighting scores, and the differences between them (i.e., attractiveness weighting minus personality weighting) produced no significant outcomes for self-monitoring, thus implying that sampling considerations did not account for the lack of self-monitoring effects in our original analyses.(6) However, the main effect for participant gender was significant in each of these ANCOVAs, all Fs $(1,41)$ [is greater than or equal to] 4.49, ps [is less than] .05, essentially replicating the findings for the sample as a whole. That is, female participants among this subsample of self-monitoring extremes weighed attractiveness information less heavily ( $\mathrm{M}=$ $55.54, \mathrm{SD}=5.25$ ) and personality information more heavily ( $\mathrm{M} 56.95, \mathrm{SD}=4.91$ ) when ranking the nine SPs than did their male counterparts $(M=59.17$ and 52.30, SDs = 6.17 and 4.90, respectively, for male attractiveness weighting and personality weighting).

Analyses of participants' top three choices. We next considered whether our results might have differed had we limited our analyses to participants' top three choices--the choices that they likely considered most important in determining with whom they would subsequently be matched. The inverse multiplier scheme described previously was once again used to determine an attractiveness weighting score and a personality weighting score for the top three choices of each participant. Each of these indexes could vary between a high of 12 and a low of 0 . These measures, and the differences between them (i.e., attractiveness weighting minus personality weighting), were then subjected to 2 (participant gender) $\times 2$ (self-monitoring) ANCOVAs, with participant attractiveness as the covariate.

Each analysis produced a now familiar and significant main effect for participant gender, all Fs $(1,95)$ [is greater than] 8.50, ps [is less than] .0 1. Even when making their top three selections, female participants weighed attractiveness cues less heavily $(M=9.09, S D=1.40)$ and personality information more heavily $(M=9.59, \mathrm{SD}=1.76)$ than did their male counterparts (Ms $=10.26$ and 8.30, SDs $=1.81$ and 1.72, respectively, for male attractiveness weighting and personality weighting). As was true for the date-ranking data for all nine SPs, male participants 
relied more heavily on attractiveness cues than on personality information when selecting their top three choices $(M=1.96, S D=2.48)$, whereas female participants relied about equally on both types of information $(M=-0.36, S D=2.73)$. None of the main effects for self-monitoring propensities nor any of the interaction terms approached significance in these analyses, all Fs $(1,95)$ [is less than] 2.00, ps [is greater than] .19.

\section{Direct Comparisons With Past Research}

We next considered our participants' reactions to the two SPs who best represented the choices offered participants in previous research. Indeed, the most direct comparison to data presented by Snyder et al. (1985) is to note the ranks that each participant assigned to the highly attractive/characterologically undesirable and the highly unattractive/ characterologic ally desirable SPs, and to then analyze the patterns of preferences that participants displayed as a function of gender and self-monitoring propensities. The resulting $2 \times 2 \times 2$ (Subject Gender $\times$ Self-Monitoring $x$ SP Preference) log-linear analysis of this relative ranking (or preference) measure produced but one significant outcome--a main effect for participant gender, [Chi square] $(N=96)=5.79, p$ [is less than] .02. As shown in the first row of Table 2, a majority of the male participants (regardless of their self-monitoring propensities) preferred the attractive/characterologically undesirable SP to her unattractive/characterologically desirable counterpart. By contrast, a majority of the female participants (even those high in selfmonitoring) preferred the unattractive/characterologically desirable SP to the attractive/characterologically undesirable individual. Although Snyder et al. (1985) ran no female participants, thus precluding direct comparisons across studies for women, we can compare the preferences of our male participants to those of men from the earlier research. The most notable difference across studies was that our low-self-monitoring men were much more inclined than those in the Snyder et al. study to prefer the attractive SP who was characterologically undesirable (i.e., $68 \%$ vs. $19 \%, z=3.35, p$ [is less than] .01 ).

\section{Modeling Participants' Use of Attractiveness Cues and Personality Information}

Recall our primary hypothesis: When evaluating prospective dates from a broad and ecologically representative array of targets, all participants should pay careful attention to both the attractiveness and the personality of such targets, seeking to maximize, as best they can, their outcomes on both dimensions. From analyses reported thus far, it might seem as if this proposition holds for women but not for men. That is, not only did men make greater use of attractiveness cues than women did when ranking the nine SPS, but the significant differences between men's attractiveness weighting scores and their personality weighting scores suggest that they had based their rankings more heavily on attractiveness cues than on personality information. Women, by contrast, appeared to rely about equally on attractiveness cues and personality information when evaluating SPs. Could it be that men's overriding concern when evaluating a prospective dating partner is the SPs physical attractiveness, whereas women are more likely than men to try to maximize their outcomes across both dimensions? To explore 
these possibilities and to test our maximization hypothesis in a more direct manner, we examined the correspondence between participants' actual rankings of the nine SPs and the rankings they should have provided had they followed each of the following ranking algorithms:

1. Attractiveness algorithm Participants using this rule would largely disregard personality information and would place (a) the three highly attractive SPs into ranks 1 to 3, (b) the three moderately attractive SPs into ranks 4 to 6, and (c) the three unattractive SPs into ranks 7 to 9.

2. Personality algorithm. Participants using this heuristic would largely disregard attractiveness cues and would place (a) the three SPs with highly desirable personalities in the first three ranks, (b) the three SPs with moderately desirable personalities in ranks 4 to 6 , and (c) the three SPs with undesirable personalities in ranks 7 to 9.

Although one can imagine other algorithms by which participants would make at least some use of both attractiveness cues and personality information to rank the nine SPS, there are two ranking heuristics that specify how SPs should have been ranked were participants consistently guided by a desire to maximize their outcomes across both dimensions. They are as follows:

3. Maximization--attractiveness bias. Participants using this rule should weigh attractiveness cues more heavily than personality when ranking SPs. However, they should be willing to make some clear attractiveness concessions to maximize outcomes across both dimensions. For example, the participant should favor a moderately attractive SP with a highly desirable personality (i.e., +1 for attractiveness, +2 for personality) over a highly attractive SP with an undesirable personality ( +2 for attractiveness, 0 for personality). Yet, whenever total outcomes across dimensions are equal, the SP who is the more attractive should be favored (e.g., a moderately attractive SP with a moderately desirable personality should be ranked higher than an unattractive SP with a highly desirable personality).

4. Maximization--personality bias. Use of this rule implies that personality is weighed more heavily than attractiveness cues when evaluating SPS. Yet, the participant should be inclined to make some clear personality concessions to maximize outcomes across both dimensions. Thus, he or she should prefer an SP with only a moderately desirable personality but who is highly attractive (i.e., +1 for personality, +2 for attractiveness) over one who has a highly desirable personality but is unattractive (+2 for personality, 0 for attractiveness). Whenever total outcomes across dimensions are equal, however, the participant should favor the SP who has the more desirable personality (e.g., an unattractive SP with a highly desirable personality over a moderately attractive SP with a moderately desirable personality).

To see how closely each participant's rankings reflected use of each of the above algorithms, we calculated Spearman rho correlations between the ranks he or she assigned to the nine SPs and the pattern of rankings anticipated by each rule. These individual correlations were then subjected to a 2 (participant gender) $\times 2$ (self-monitoring) $\times 4$ (algorithms) repeated-measures ANOVA (with algorithms as the repeated factor) to determine (a) with which rating algorithms participants' rankings were most consistent and (b) whether the correspondence between participant rankings and rating algorithms varied as a function of participant gender and selfmonitoring propensities. 
The ANOVA produced but two significant outcomes. A main effect for algorithms, $F(3,279)=$ $47.89, \mathrm{p}$ [is less than] .001, reflected the finding that participants' rankings corresponded more closely to the use of either of the maximization algorithms (mean is $=.75$ for both maximization-attractiveness bias and maximization--personality bias algorithms) than to rankings predicted by the simple attractiveness algorithm (mean $r=.50), F(1,239)=5.32$, $p$ [is less than] .05 , or the simple personality algorithm (mean $r=.49) \mathrm{F}(1,239)=5.53$, $\mathrm{p}$ [is less than] .05 . In other words, it appears as if our participants were generally attempting to maximize outcomes across dimensions rather than basing their rankings on predominantly one dimension or the other.(7)

However, the main effect for algorithms was qualified by a two-way interaction between participant gender and algorithms, $F(3,279)=13.87, \mathrm{p}$ [is less than] .001 . Simple effects analyses revealed that the simple main effect of participant gender was significant for three of the four algorithms: the simple attractiveness rule, $F(1,95)=6.23, p$ [is less than] .05; the simple personality rule, $F(1,95)=11.53$, $p$ [is less than] .01; and the maximization--personality bias rule, $F(1,95)=8.31, p$ [is less than] .01 . As shown in Table 3 , the date rankings of male participants corresponded more closely than those of female participants to those expected by the simple attractiveness rule. By contrast, the rankings of female participants corresponded more closely than those of male participants to both the simple personality algorithm and the maximization--personality bias algorithm.

TABLE 3: Mean Correlations Between the Ranks Assigned to Nine Stimulus Persons by Male and Female Participants and the Patterns of Rankings Anticipated by Four Ranking Algorithms

Rating Algorithm

\begin{tabular}{|c|c|c|}
\hline $\begin{array}{l}\text { Subject } \\
\text { Gender }\end{array}$ & Attractiveness & Personality \\
\hline \multicolumn{3}{|l|}{ Male } \\
\hline$M$ & [.57.sub.c] & [.38.sub.d] \\
\hline SD & .26 & .29 \\
\hline \multicolumn{3}{|l|}{ Female } \\
\hline$M$ & [.42. sub.d] & [.58.sub.c] \\
\hline \multirow[t]{3}{*}{ SD } & .26 & .28 \\
\hline & \multicolumn{2}{|c|}{ Rating Algorithm } \\
\hline & $\begin{array}{l}\text { Maximization- } \\
\text { Attractiveness }\end{array}$ & $\begin{array}{l}\text { Maximization } \\
\text { Personality }\end{array}$ \\
\hline Gender & Bias & Bias \\
\hline \multicolumn{3}{|l|}{ Male } \\
\hline M & [.78.sub.a] & [.71.sub.b] \\
\hline SD & .18 & .16 \\
\hline \multicolumn{3}{|l|}{ Female } \\
\hline$M$ & {$[.73$. sub.ab] } & [.79. sub.a] \\
\hline SD & .12 & .13 \\
\hline
\end{tabular}

NOTE: Each mean correlation is based on an $\mathrm{n}$ of 50 . Means with common subscripts are not significantly different at $\mathrm{p}$ [is less than] .05 . 
Thus, it once again appears as if men were more attractiveness oriented when ranking SPs than women were. And yet, men also displayed a strong interest in the dispositional attributes of prospective dating partners, as evidenced by the observation that the maximization-attractiveness bias algorithm represented the best approximation of the ratings men provided (see Table 3). By contrast, women were more personality oriented when judging the nine SPs than men were. Nevertheless, their concurrent interest in attractiveness cues is quite apparent from the findings that the maximization--personality bias algorithm represented the best (absolute) approximation of the female-date rankings, a correspondence that was significantly greater than that emerging for the simple personality rule.(8)

\section{DISCUSSION}

We began this project seeking to determine whether the systematic differences in dating orientations reported for high- versus low-self-monitoring individuals in past research (e.g., Glick, 1985; Glick et al., 1988; Snyder et al., 1985) might not have been overdramatized. Our bases for this suspicion were primarily methodological ones. Prior research has consistently indicated that high-self-monitoring individuals focus heavily on external appearances (i.e., physical attractiveness) and that low-self-monitoring monitoring individuals attend more closely to dispositional information (i.e., personality) when selecting dating partners. However, the most dramatic evidence for these claims comes from studies (e.g., Glick, 1985; Snyder et al., 1985, Experiment 2) in which participants were constrained to choose between two opposite-sex targets for whom level of attractiveness and characterological desirability varied extremely and were perfectly confounded (i.e., a highly attractive SP who was characterologically undesirable vs. a highly unattractive SP with a very desirable personality). Of course, such a decision requires participants to sacrifice one desirable attribute (e.g., attractiveness) for another (e.g., a desirable personality) and, thus, may seriously overestimate the extent to which they would rely on this preferred dimension to evaluate prospective dating partners in the natural ecology (in which attractiveness cues and personality information are not so confounded, and such extreme sacrifices may rarely, if ever, be called for). Moreover, the existing research has focused on the dating orientations of high- and low-self-monitoring men--a potentially important limitation in view of the burgeoning evidence (cf. Buss, 1989; Feingold, 1990, 1992) that women are more inclined than men to focus closely on demographic and dispositional information and somewhat less intently on attractiveness cues when evaluating romantic partners.

Our purpose, then, was to determine just how reliable the different dating orientations of highand low-self-monitoring individuals would prove to be when participants were asked to evaluate prospective dating partners drawn from a broader, more representative array of opposite-sex targets in a situation in which levels of physical attractiveness and desirability of personality information were orthogonally varied. Clearly, we would not have been surprised to find some variation in date rankings as a function of self-monitoring propensities--evidence that would possibly be stronger for male participants than for females. Yet, we also presumed that virtually all of our participants would carefully consider all kinds of information provided to them about 
prospective dating partners and would make decisions that would serve to maximize their total outcomes across both the personality and the attractiveness dimensions.

\section{Findings for Self-Monitoring}

The aspect of our results that surprised us most was the paucity of evidence for any differences in the dating orientations of high- and low-self-monitoring individuals. Regardless of whether the analyses focused on participants' top three choices (i.e., the SPs with whom participants were led to believe they would soon meet and could be paired) or on their evaluations of the entire array of nine SPs, high-self-monitoring individuals were not more inclined than their low-selfmonitoring counterparts to overweight attractiveness cues or to underweight dispositional information. Moreover, the SP rankings provided by high- and low-self-monitoring participants did not vary with respect to their degree of correspondence with rankings anticipated by either a simple attractiveness or a simple personality rating algorithm. But as anticipated by our alternative maximization hypothesis, the rating algorithms that provided the best fit with the rankings of both high- and low-self-monitoring individuals were those implying that participants had relied on both attractiveness and dispositional information and had attempted to maximize their outcomes across both these dimensions (i.e., for high-self-monitoring individuals, mean rs $=.76$ and .73, respectively, for maximization--attractiveness bias and maximization--personality bias algorithms; for low-self-monitoring individuals, mean rs $=.75$ and. 77 , respectively, for the maximization--attractiveness bias and maximization--personality bias algorithms).(9)

Of course, no one (to our knowledge) has ever claimed that the presumed attractiveness orientation of high-self-monitoring individuals or the dispositional bias of their low-self-monitoring counterparts are all-or-nothing criteria for decision making. Snyder et al. (1985) viewed these contrasting foci as judgmental "priorities" that are broad based and should be detectable in freechoice settings (e.g., Snyder et al., 1985, Experiment 1, or in scenarios such as our own) but that are likely to be stronger whenever a person must make a "hard choice" (i.e., sacrifice one desirable attribute for another). In fact, Snyder et al. (Experiment 2) purposely confounded the attractiveness and the characterological desirability of their two SPs to present their subjects with just such a choice. Yet, our own data caused us to question earlier interpretations of the Snyder et al. (Experiment 2) results. Consider first that we were unable to replicate the hard choice data reported by Snyder et al.--that high-self-monitoring individuals would clearly prefer a highly attractive/characterologically undesirable SP to a highly unattractive/characterologically desirable one, whereas low-self-monitoring individuals would display the opposite preferential pattern (see Table 2). Admittedly, this replication failure may stem, in part, from the fact that our participants had seven additional degrees of freedom against which to compare these extreme alternatives and, thus, were not constrained to select one of these two SPs as their date. Nevertheless, these two SPs were assigned very similar (rather than dissimilar) mean ranks by both high- and low-self-monitoring individuals (i.e., for high-self-monitoring participants, Ms = 5.45 and 5.56, respectively, for the highly attractive and the characterologically desirable SPS; for low-self-monitoring participants, Ms $=5.62$ and 5.36, respectively, for the highly attractive and characterologically desirable options), a finding anticipated by the maximization of 
outcomes hypothesis and offering little support for the notion that dating orientations vary dramatically as a function of self-monitoring propensities. Second (and equally important for interpretive purposes), the low mean ranks that these two SPs attained imply that participants viewed these people as relatively undesirable prospective dates. Thus, if high-and low-selfmonitoring participants in the Snyder et al. study (Experiment 2) had similar reservations about the two (conceptually identical) alternatives presented to them, their contrasting preferences may not have reflected their dating orientations (i.e., approach tendencies) at all but, rather, their strategies for cutting their losses when forced to make a distasteful dating decision of the kind they may rarely face and would ordinarily seek to avoid.

Consistent with this logic, not one of our participants selected the attractive/characterologically undesirable or the unattractive/characterologically desirable SPs as one of their top three choices, with whom it was likely that they would be paired. Indeed, every high and low selfmonitor in this experiment was an absolute maximizer in the sense of having selected the highly attractive individual with the highly desirable personality as the top-ranked person with whom he or she hoped to be matched. So even though participants in this study may have had some uncertainty about which of two or three SPs they might be paired with for an evening date, their unanimity with respect to a top choice implies that they were taking their rankings seriously and were trying, as best they could, to ensure that they would be exposed to that individual who offered them most in terms of attractiveness and desirable personality characteristics.

In sum, our results provide little evidence for any major differences in the criteria used by highand low-self-monitoring individuals when selecting and evaluating prospective dating partners from a broadly representative array of alternatives. Indeed, both groups of participants relied heavily on attractiveness cues and dispositional information, and they appeared to prefer as prospective dates those SPs who promised to maximize their total outcomes across both dimensions.

Finally, a methodological note. Previous work on self-monitoring and dating preferences has used the original 25-item Self-Monitoring Scale (SMS; see Snyder, 1987), whereas we assessed self-monitoring propensities with the newer 18-item SMS. This 18-item scale was created in response to reports that the 25-item SMS was multifactorial (e.g., Briggs, Cheek, \& Buss, 1980), and it was said to be a more factorially pure measure of the self-monitoring construct (Snyder, 1987). However, the latter assertion has been challenged by research revealing the 18item SMS consists of two factors that had emerged from analyses of the 25item SMS--Public Performing and Other-Directedness (e.g., Briggs \& Cheek, 1988). Our failure to replicate the earlier dating research is not, in our opinion, a measurement issue in that the 18item SMS is a strong embodiment of the Public Performing factor (cf. John, Cheek, \& Klohnen, in press) and the attendant desire to manage impressions that are said to underlie alleged differences in the dating orientations of high and low self-monitors (Snyder, 1987). Unfortunately, however, the Other-Directedness component of self-monitoring is less well represented in the 18-item SMS, as compared with the 25-item SMS (John et al., in press). Thus, we recommend that future research addressing the romantic inclinations of high- an lowself-monitoring individuals employ the earlier 25-item SMS and explore Public Performing and 
Other-Directedness as potential moderators of whatever results might emerge as a function of overall self-monitoring propensities.

\section{Findings for Gender}

The only group differences in date rankings that we were able to detect were those that emerged for men versus women. Regardless of whether the analyses centered on participants' top three choices or on their ordering of the entire array of nine SPs, the rankings that men provided were influence more attractiveness cues and less by dispositional information than were those provided by women. On an absolute basis, men relied more heavily on attractiveness cues than on personality information when ranking the nine SPs, and, indeed, their rankings corresponded more closely to those expected by a simple attractiveness rating algorithm than to the pattern anticipated by a simple personality algorithm. By contrast, women weighed attractiveness cues and dispositional information about equally, although their rankings of the nine SPs corresponded more closely to those anticipated by the personality algorithm rather than the attractiveness algorithm. Taken together, these outcomes dovetail nicely with conclusions drawn in past research regarding the criteria used by men and women to evaluate romantic partners (cf. Buss, 1989; Feingold, 1990, 1992).

And yet, it would be inappropriate, we believe, to use either the present or past findings to tag men as attractiveness centered or women as dispositionally focused in their dating orientations. Why? Because participants of each gender ranked SPs in ways implying that they were striving to maximize their total outcomes across o e attractiveness and personality dimensions. Male participants did appear to favor a maximization rule that gave slightly more weight to attractiveness cues than to dispositional information, whereas the other maximization rule (i.e., the maximization--personality bias algorithm) provided the best approximation of the pattern of rankings provided by female participants. Although these best-fitting correlations were far from perfect for either the male or the female participants (mean $n=+.78$ and +.79 , respectively),(10) they nonetheless accounted for significantly more variability in the date rankings than either the simple attractiveness or personality algorithms--rules that come closer to representing truly attractiveness-oriented and dispositionally focused dating orientations.

Recently, Landolt, Lalumiere, and Quinsey (1995) orthogonally varied the physical attractiveness and the earning potential of prospective dating partners and have reported results similar to our own. Specifically, both men and women clearly preferred to date those SPs who were both highly attractive (rather than moderately attractive or unattractive) and high (rather than low) in earning potential. Thus, participants of each sex were apparently striving to maximize outcomes across both the attractiveness and the demographic dimensions, rather than weighing either dimension notably higher than the other. The only reliable sex difference in ratings of prospective dating partners was the finding that women gave more weight to earning potential than men did when evaluating the desirability of highly attractive (but not moderately attractive or unattractive) SPs. 
In sum, men and women may indeed display slightly different priorities when evaluating the desirability of prospective dating partners who vary in attractiveness and

personality/demographic characteristics. And although additional research may be necessary to resolve the issue, we are hesitant to conclude that these gender-linked priorities represent qualitatively distinct dating orientations. Women's rankings did correspond more closely than those of men to the pattern specified by the maximization--personality bias algorithm. However, the correspondence between participants' date rankings and the pattern anticipated by the maximization--attractiveness bias algorithm did not differ by gender. Thus, our most prudent conclusion might be that men and women display dating orientations that are really more similar than they are different: Both the looks and the character of prospective dating partners are highly salient to men and women, who seem most interested in pursuing relationships with those individuals who offer them most across both these dimensions.

\section{NOTES}

(1.) Snyder et al. (1985) defended this reliance on male participants by arguing that it is men who still initiate most dates in our society. Yet, this reasoning overlooks the fact that women must still decide whether to acceptor reject a dating invitation and, thus, have ample opportunity to allow attractiveness cues and dispositional information to influence the decisions they make.

(2.) In addition, the three SPs of each gender at each level of attractiveness did not differ in their perceived physical attractiveness (all Fs [is less than] 1, ns).

(3.) Moreover, persons described by the three profiles at each desirability level did not differ with respect to perceived likability (all Fs [is less than] 1, ns).

(4.) To illustrate, an attractiveness weighting score of 63 would result had the participant selected highly attractive SPs for ranks 1 to 3, moderately attractive SPs for ranks to 4 to 6 , and unattractive SPs for ranks 7 to 9. By contrast, selecting unattractive SPs for ranks 1 to 3 , moderately attractive SPs for ranks 4 to 6, and highly attractive SPs for ranks 7 to 9 would yield an attractiveness weighting score of 27.

(5.) Analogous attractiveness weighting and personality weighting scores collected form pretest participants in a sophomore-level laboratory course were highly stable over a 10- to 14-day period, rs (22) $=.92$ and .90 , respectively, for attractiveness weighting and personality weighting).

(6.) The only outcome involving the self-monitoring variable that even approached significance in these analyses was a marginal main effect of self-monitoring for the personality weighting measure. Extremely high self-monitoring individuals weighed personality information somewhat less heavily $(M=53.07)$ when ranking the nine SPs than did their extremely low self-monitoring counterparts $(M=56.53), F(1,41)=3.03, p=.09$.

(7.) This conclusion stems not only from the above analyses but also from correlations we computed between participants' date rankings and patterns anticipated by other rating 
heuristics--algorithms by which participants might have used both attractiveness and personality information without the stipulation that they maximize outcomes across both dimensions (e.g., grouping SPs into three attractiveness categories and then ranking the three SPs within each category by desirability of personality). The resulting correlations often varied by gender (though not by self-monitoring propensities). However, the correlations only ranged between +.41 and +.54 and represented no significant improvement in predictability beyond that provided by the simple attractiveness and simple personality algorithms. Thus, we are confident in concluding that the maximization algorithms were, indeed, the closest approximations of the strategies our participants followed when ranking the nine SPs.

(8.) Correlations between the average ranks that male and female participants assigned to each of the nine SPs and the rankings anticipated by each rating algorithm were quite consistent with the above findings. For example, the average ranks assigned to particular SPs by male participants were very closely aligned with those anticipated by the maximization--attractiveness bias algorithm, $r(8)=.96$, with the other algorithms providing somewhat poorer fits with these data (i.e., maximization--personality bias, $r=.82$; attractiveness, $r=.62$; personality, $r=.42$ ). By contrast, average rankings of individual SPs by female participants came closer to those anticipated by the maximization--personality bias algorithm, $r(8)=.92$, than to any other rule (i.e., maximization--attractiveness bias, $r=.83$; personality, $r=.69$; attractiveness, $r=.44$ ).

(9.) Although we tested more than three times the number of participants Snyder et al. (1985, Experiment 2) did in their research, one might nonetheless wonder whether we lacked sufficient power to detect any systematic differences in the date rankings of high- and low-self-monitoring individuals. Using the original data of Snyder et al. (1985, Experiment 2) to estimate a large selfmonitoring effect and halving these differences twice more to estimate medium and small effects, we calculated our power at better than $90 \%$ for detecting large and medium variations in algorithm matching as a function of self-monitoring propensities and at better than $85 \%$ for detecting a small variation. Thus, it is highly unlikely that our failure to find systematic differences in the date rankings of high- and low-self-monitoring individuals reflects a Type II error.

(10.) Personal idiosyncrasies (i.e., the tendency of individual perceivers, based on personal tastes, to over- or underestimate the desirability of particular personality profiles or the attractiveness of particular faces) undoubtedly conspired to attenuate these correlations somewhat. Yet, if we can assume that any such idiosyncratic judgmental biases are randomized across participants and SPs, then the mean rank that participants assigned to a particular SP should cancel the effects of these biases on the evaluation of that individual. This is the reason we calculated the average rank/algorithm correlations reported in note 7--correlations that imply that the maximization--attractiveness bias algorithm $(r=+.96)$ and the maximization--personality bias algorithm $(r=+.92)$ were very close approximations of the strategies used by men and women, respectively, when ranking the nine SPs. 


\section{REFERENCES}

Anderson, N. H. (1968). Likableness ratings of 555 personality-trait words. Journal of Personality and Social Psychology, 70, 394-400.

Bazzini, D. G., \& Shaffer, D. R. (1995). Investigating the social-adjustive and value-expressive functions of well-grounded attitudes: Implications for change and for subsequent behavior. Motivation and Emotion, 19, 279-305.

Briggs, S. R., \& Cheek, J. M. (1988). On the nature of self-monitoring: Problems with assessment, problems with validity. Journal of Personality and Social Psychology, 54, 663-678.

Briggs, S. R., Cheek, J. M., \& Buss, A. H. (1980). An analysis of the Self-Monitoring Scale. Journal of Personality and Social Psychology, 38, 679-686.

Buss, D. M., (1989). Sex differences in human mate preferences: Evolutionary hypotheses tested in 37 cultures. Behavioral and Brain Sciences, 12, 1-49.

Buss, D. M., \& Schmitt, D. P. (1993). Sexual strategies theory: An evolutionary perspective on human mating. Psychological Review, 100, 204-232.

Cloninger, S. C. (1996). Personality: Description, dynamics, and development. New York: Freeman.

Feingold, A. (1990). Gender differences in effects of physical attractiveness on romantic attraction: A comparison across five research paradigms. Journal of Personality and Social Psychology, 59, 981-993.

Feingold, A. (1992). Gender differences in mate selection preferences: A test of the parental investment model. Psychological Bulletin, 112, 125-139.

Fischer, J. M., \& Heesacker, M. (1995). Men's and women's preferences regarding sex-related and nurturing traits in dating partners. Journal of College Student Development, 36, 260-269.

Glick, P. (1985). Orientations toward relationships: Choosing a situation in which to begin a relationship. Journal of Experimental Social Psychology, 21, 544-562.

Glick, P., De Morest, J. A., \& Hotze, C. A. (1988). Self-monitoring and beliefs about partner compatibility in romantic relationships. Personality and Social Psychology Bulletin, 14, 485-494.

Graziano, W. G., Jensen-Campbell, L. A., Shebilske, L. J., \& Lundgren, S. R. (1993). Social influence, sex differences, and judgments of beauty: Putting the interpersonal back in interpersonal attraction. Journal of Personality and Social Psychology, 65, 522-531.

Hatfield, E., \& Rapson, R. L. (1996). Love and sex: Cross-cultural perspectives. Boston: Allyn \& Bacon.

Horowitz, I. A., \& Bordens, R. S. (1995). Social psychology. Mountain View, CA: Mayfield. 
Jensen-Campbell, L. A., Graziano, W. G., \& West, S. G. (1995). Dominance, prosocial orientation, and female preferences: Do nice guys really finish last? Journal of Personality and Social Psychology, 68, 427-440.

John, O. P., Cheek, J. M., \& Klohnen, E. C. (in press). On the nature of self-monitoring: Construct explication via Q-sort ratings. Journal of Personality and Social Psychology.

Landolt, M. A., Lalumiere, M. L., \& Quinsey, V. L. (1995). Sex differences in intra-sex variations in human mating tactics: An evolutionary approach. Ethology and Sociobiology, 16, 3-23.

Myers, D. G. (1996). Social psychology (5th ed.). New York: McGraw-Hill.

Snyder, M. (1987). Public appearances/private realities: The psychology of self monitoring. New York: Freeman.

Snyder, M., Berscheid, E., \& Glick, P. (1985). Focusing on the exterior and the interior: Two investigations of the initiation of personal relationships. Journal of Personality and Social Psychology, 48, 1427-1439.

Snyder, M., \& Gangestad, S. (1986). On the nature of self-monitoring: Matters of assessment, matters of validity. Journal of Personality and Social Psychology, 51, 125-139.

Snyder, M., \& Simpson, J. A. (1984). Self-monitoring and dating relationships. Journal of Personality and Social Psychology, 47, 1281-1291.

Trivers, R. L. (1972). Parental investment and sexual selection. In B. Campbell (Ed.), Sexual selection and the descent of man 1871-1971 (pp. 136-172). Chicago: Aldine. 